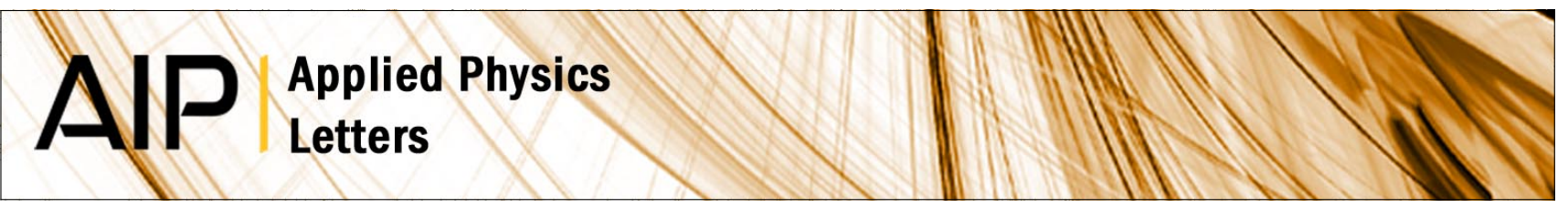

\title{
Production of self-aligned metal nanocolumns embedded in an oxide matrix film
}

Jérémie Margueritat, Jose Gonzalo, Carmen N. Afonso, M. Isabel Ortiz, and Carmen Ballesteros

Citation: Appl. Phys. Lett. 88, 093107 (2006); doi: 10.1063/1.2180877

View online: http://dx.doi.org/10.1063/1.2180877

View Table of Contents: http://apl.aip.org/resource/1/APPLAB/v88/i9

Published by the American Institute of Physics.

Additional information on Appl. Phys. Lett.

Journal Homepage: http://apl.aip.org/

Journal Information: http://apl.aip.org/about/about_the_journal

Top downloads: http://apl.aip.org/features/most_downloaded

Information for Authors: http://apl.aip.org/authors

\section{ADVERTISEMENT}

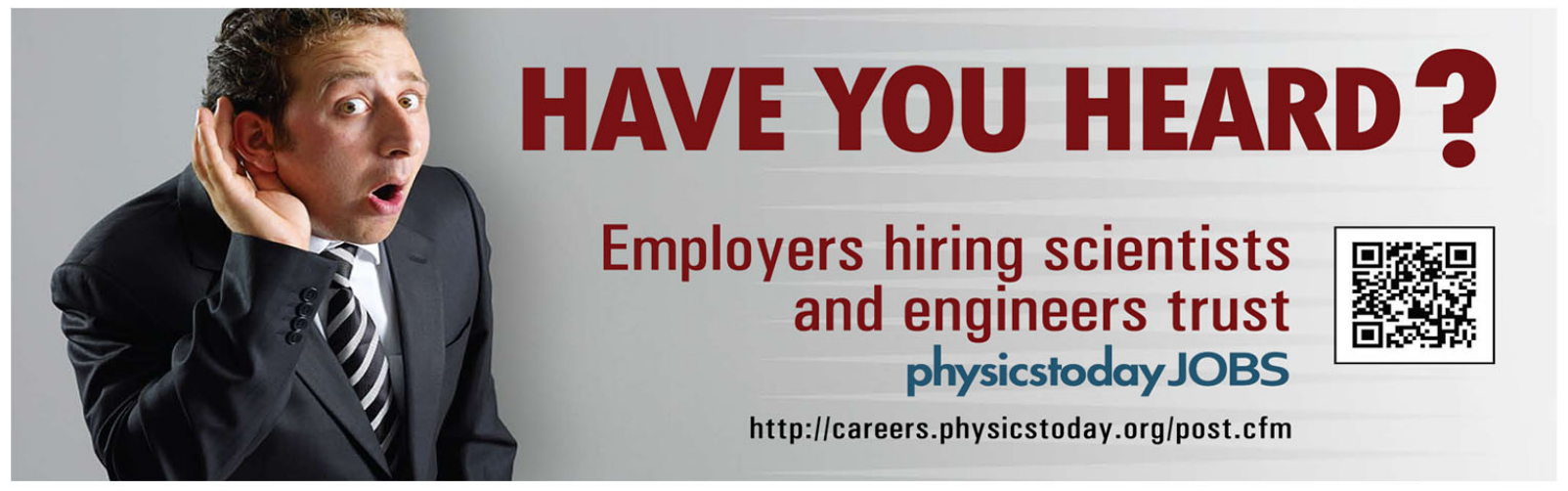




\title{
Production of self-aligned metal nanocolumns embedded in an oxide matrix film
}

\author{
Jérémie Margueritat, ${ }^{\text {a) }}$ Jose Gonzalo, and Carmen N. Afonso \\ Laser Processing Group, Instituto de Optica, CSIC, Serrano 121, 28006 Madrid, Spain \\ M. Isabel Ortiz and Carmen Ballesteros \\ Laboratorio de Microscopía Electrónica de Transmisión, LABMET, Universidad Carlos III, Av. Universidad \\ 30, 28911 Leganés, Madrid, Spain
}

(Received 9 August 2005; accepted 18 January 2006; published online 28 February 2006)

\begin{abstract}
Self-aligned silver nanocolumns embedded in amorphous $\mathrm{Al}_{2} \mathrm{O}_{3}$ matrix are produced by alternate pulsed laser deposition at room temperature in a single step process. The morphology and the spatial distribution have been investigated by standard, high resolution, and scanning transmission electron microscopy. Polycrystalline nanocolumns having diameters close to $3 \mathrm{~nm}$ and heights up to $7 \mathrm{~nm}$ are all oriented perpendicular to the substrate surface. The self-alignment of the nanocolumns is the consequence of the deposition of consecutive layers of $\mathrm{Ag}$ nanoparticles using the first layer as template for the ordered growth of the nanocolumns. Control over the density, diameter and height of the nanocolumns is achievable by tailoring the deposition sequence. (C) 2006 American Institute of Physics. [DOI: 10.1063/1.2180877]
\end{abstract}

Metallic nanostructures have a high potential for the production of nanoscale electronic, optoelectronic or magnetic devices. Examples of this potential are the use of nanostructures as building blocks for chemical sensors, ${ }^{1}$ substrates for surface enhanced Raman scattering, ${ }^{2-4}$ and contacts $^{5}$ or single-electron transistors ${ }^{6}$ for nanoelectronics. In this respect, intense efforts have been focused on the production of metal nanorods and nanowires due not only to their essential role in the fabrication of new devices but also because they offer opportunities to study key fundamental aspects such as quantized conductance or new plasmonic effects. Bulk silver exhibits the highest electrical and thermal conductivity among all metals and silver nanoparticles of different sizes and shapes exhibit surface plasmon resonances that can be well resolved over interband transitions and tuned in the visible spectrum range. It is thus a very suitable metal case for studying both the fundamental and technological aspects of elongated and oriented nanostructures and there have thus been several reports on the production of silver nanorods or nanowires. $^{7-14}$ Different methods have been developed to prepare such metal elongated nanostructures most of them using chemical routes (thermal, ${ }^{8}$ hydrothermal, ${ }^{10}$ or oxide reduction, ${ }^{1,7,11,12,14}$ ) or using templates (i.e., mesoporous silica $^{9}$ or alumina $\left.{ }^{3,4}\right)$. They generally lead to randomly oriented nanowires that have a diameter smaller than $100 \mathrm{~nm}$, lengths of a few micrometers or longer, and are in most cases in colloidal suspensions. However, for many of the envisaged applications, these elongated nanostructures need to be oriented and supported on substrates or embedded in matrices. Another important challenge that needs to be faced is the control of the shape and dimension dispersion in order to keep it low enough. There have been some works reporting contributions in this direction using multi-step approaches based on the use of electron beam lithography. $2,6,9,13,15$

In this letter, we report a new single step method using pulsed laser deposition (PLD) to produce oriented Ag nano-

\footnotetext{
a) Author to whom correspondence should be addressed; electronic mail:
} j.margueritat@io.cfmac.csic.es wires embedded in a matrix and having low dimension and shape dispersion. PLD has already been proven to be very suitable for the growth of complex oxide films. ${ }^{16}$ It is based on focusing nanosecond laser pulses from a laser onto a target material typically in the ultraviolet (UV) and having nanosecond pulse duration. The use of two independent targets of an oxide (for the matrix) and of a metal have successfully been applied to produce high quality nanocomposite films formed by metal nanoparticles (NPs) embedded in amorphous aluminium oxide $\left(a-\mathrm{Al}_{2} \mathrm{O}_{3}\right) \cdot{ }^{17,18}$ Figure 1 shows cross section schematics of one of those films that consist of "layers" of metal NPs of diameter $\mathbf{D}$ and whose spacing can easily be controlled through the deposition of the matrix, $\mathbf{X}$ being the equivalent thickness of the matrix layer. The technique for the deposit of the nanowires is an extension of this approach since it is based on the deposition of a limited amount of the matrix by decreasing $\mathbf{X}$ down to values close to $\mathbf{D}$. The matrix acts then as a template that allows an oriented growth of the nanowires and the sequence is repeated a number of times $n$ to lead to the situation sketched in Fig. 1. The resulting nanowires are oriented perpendicular to the substrate and the method allows to deliberately selecting their length through the number of cycles. Both features are not easily accessible via the chemical methods cited above or require a multistep approach such as in the case of lithography based techniques.

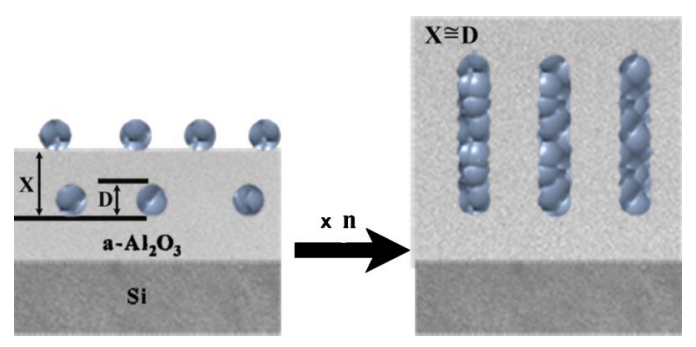

FIG. 1. (Color online) Schematics for the films growth and deposition sequence to produce $\mathrm{NCls}$. 
An ArF excimer laser [full width at half maximum (FWHM) pulse duration $=20 \mathrm{~ns}$, repetition rate of 5 and $20 \mathrm{~Hz}$ for the $\mathrm{Ag}$ and the $\mathrm{Al}_{2} \mathrm{O}_{3}$ targets, respectively], with an average energy density of $1.9 \mathrm{~J} \mathrm{~cm}^{-2}$, was sequentially focused on the surface of high purity $\mathrm{Al}_{2} \mathrm{O}_{3}$ and $\mathrm{Ag}$ rotating targets. The films were grown in vacuum $\left(10^{-6} \mathrm{mbar}\right)$ on rotating substrates held at room temperature placed $32 \mathrm{~mm}$ away from the target surface. The films are produced on $\mathrm{Si}$ substrates covered by their native oxide held at room temperature. From the deposition rates of $\mathrm{Ag}$ and the $\mathrm{Al}_{2} \mathrm{O}_{3}$ required to produce NPs of a certain diameter and matrix layers of a defined thickness, already known from earlier works, ${ }^{17,18}$ we have selected our experimental conditions to produce nanowires with typical dimensions of an average diameter $\mathbf{D} \cong 2 \mathrm{~nm}$ and a matrix layer thicknesses ranging from $\mathbf{X}=2 \mathbf{D}$ to $\mathbf{X}=0.5 \mathbf{D}$. The number of cycles was fixed to 5 , and thus the produced nanostructures have a small aspect ratio when compared to the nanostructures typically referred to as nanowires in the literature. This feature together with the fact that they are all oriented along the direction perpendicular to the substrate, make the present nanostructures to be nanocolumns $(\mathrm{NCls})$ rather than nanowires.

The morphology and structure of the films have been studied by transmission electron microscopy in a Philips Tecnai 20F FEG, transmission electron microscopy (TEM)/ scanning TEM (STEM) analytical microscope operating at $200 \mathrm{kV}$ with point-to-point resolution of $0.2 \mathrm{~nm}$ and equipped with an electron dispersive X-ray (EDX) detector, and a dark-field high angle annular detector for $Z$-contrast analysis. Composition mapping was performed in STEMEDX mode. Both plan view and cross-sectional specimens have been prepared by mechanical polishing followed by ion milling in a liquid-nitrogen cooled holder at $3 \mathrm{kV}$ and final angle of $8^{\circ}$. The silicon substrate in the cross section specimens has been used to perfectly orient the specimen perpendicular to the electron beam as well as to calibrate the scale of the images.

Figure 2 shows cross-section TEM images of films produced with different $\mathbf{X}$ values. When $\mathbf{X}=2 \mathbf{D}$, no columnar growth is expected to occur as it is indeed seen in the image. Instead, it shows the excellent control achieved in the growth process by PLD since 5 dark "layers" of NPs can be well resolved. The average diameter of the NPs (D $\sim 1.9 \pm 0.2 \mathrm{~nm}$ ) as well as the spacing of the NPs "layers" $(\mathbf{X} \sim 5.0 \pm 0.2 \mathrm{~nm})$ agrees well with what was intended. When $\mathbf{X}=\mathbf{D}$, elongated dark areas perpendicular to the substrate and thus oriented along the growth direction are observed. Figure 2 also shows that these elongated structures are well separated one from another for $\mathbf{X} \cong \mathbf{D}$ and oriented perpendicular to the substrate. The length or height $\mathbf{H}$ of these elongated structures is plotted in Fig. 3 as a function of $\mathbf{X}$, where it seems that $\mathbf{H}$ undergoes a maximum of $7 \pm 0.2 \mathrm{~nm}$ for $\mathbf{X}$ in the neighborhood of $\mathbf{D}$. Figure 3 also shows a high resolution image of one of the NCls obtained for $\mathbf{X}=\mathbf{D}$ where lattice fringes are observed evidencing their crystalline structure. Fourier transform patterns of the NCls indicate that they are not single crystals. This is also seen in Fig. 3 where regions having fringes with different orientations along the $\mathrm{NCl}$ are clearly visible.

Figure 4 shows plan view images of the specimen grown with $\mathbf{X}=\mathbf{D}$ taken in TEM and STEM mode with EDX analysis for X-rays and high angle annular detector for Z-contrast images, the latter showing the areas having large concentra-
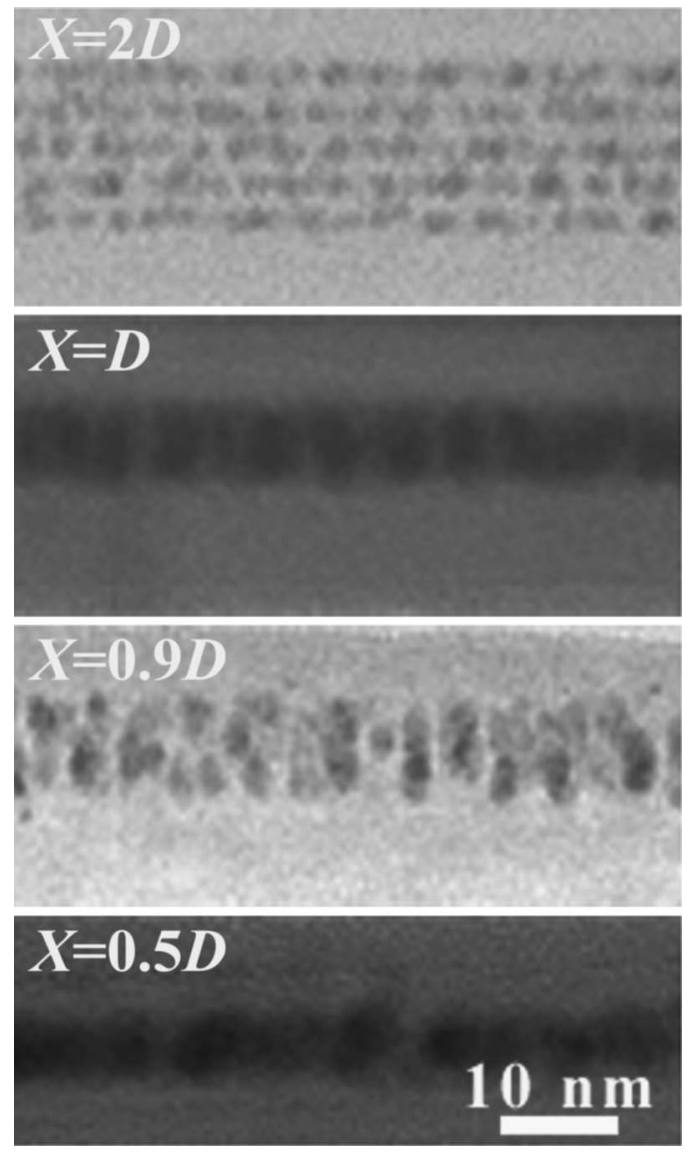

FIG. 2. Cross-sectional TEM images of films produced with different effective thickness of the matrix layer $\mathbf{X}$ with respect to the diameter $\mathbf{D}$ of metal NPs.

tions of $\mathrm{Ag}$ as bright areas. The good match of dark and bright areas in both images together with the X-ray fluorescence spectra confirms that isolated silver NCls have been produced in the direction perpendicular to the substrate. The $\mathrm{NCls}$ have an approximately round section with a very homogenous diameter of $2.7 \pm 0.2 \mathrm{~nm}$ as it shown in the histogram included in Fig. 4.

When depositing a metal on an oxide surface, it is well known that it tends to follow the Volmer-Weber threedimensional growth due to the lower surface free and interface energies of the metal with respect to that of the substrate. In addition, pulsed laser deposition (PLD) is a process involving a large ion to neutral species ratio and it is also well known that the presence of such charged species creates nucleation centers at the substrate. ${ }^{19}$ A high number density of NPs are thus homogenously nucleated over the whole sub-

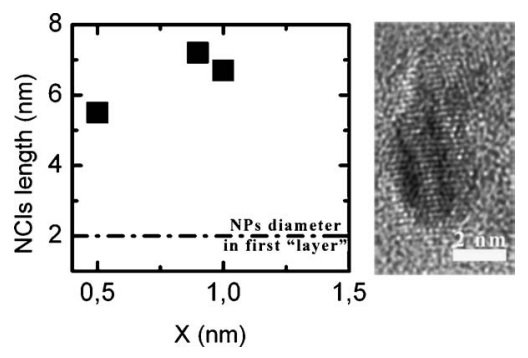

FIG. 3. Length $\mathbf{H}$ of NCls as a function of the effective thickness of the matrix layer $\mathbf{X}$ and high-resolution cross-section TEM image of one of the $\mathrm{NCl}$ produced with $\mathbf{X}=\mathbf{D}$. 

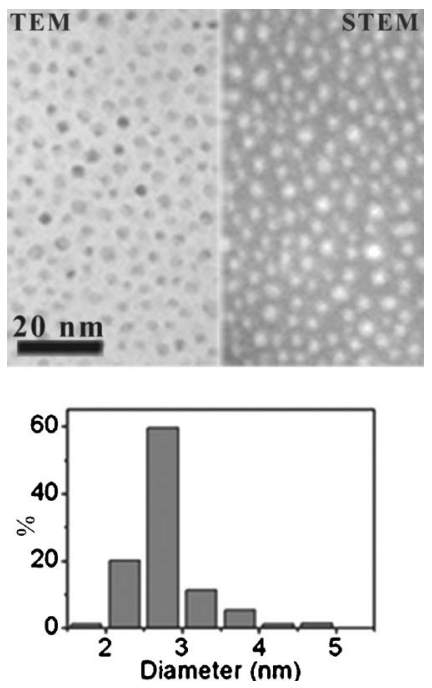

FIG. 4. TEM and STEM plan view images of the specimen produced with $\mathbf{X}=\mathbf{D}$, the latter being imaged with Ag signal. The plot includes the diameter dispersion measured in the TEM image.

strate at the early stages leading to NPs dimensions close to $2 \mathrm{~nm}$ for the case of Ag (Ref. 17) or Au (Ref. 18) before coalescence starts. This stage is illustrated in Fig. 2 for $\mathbf{X}$ $=2 \mathrm{D}$. To generate NCls, the production of this first "layer" of metal NPs is followed by the deposition of a certain amount of the matrix material, enough to fill-in the space among the NPs while leaving uncovered part of the metal NPs. The matrix thus acts as a mask defining a template where the next ablated metal self-organizes driven by the higher metal-metal than metal-oxide adhesion energy and the high mobility of the atoms at the surface due to the high kinetic energies involved in the PLD process. ${ }^{20}$ This self-organization process leads to the production of $\mathrm{NCls}$ when the process is repeated a number of times. The first layer of NPs constitutes thus the seeds for the NCls. Since it has been reported that the number density of NPs decreases as the amount of metal deposited per layer increases due to coalescence, ${ }^{19}$ the number density of NCls, their diameter and thus, their separation, can potentially be controlled by the amount of metal in this first layer of NPs. Since previous results on the synthesis of Ag NPs (Ref. 17) show that NPs with diameters in the range of $2 \mathrm{~nm}$ to $5 \mathrm{~nm}$ can be produced at room temperature before the onset of coalescence, the NCls achievable diameter is most likely in the same range. The thickness $\mathbf{X}$ of the matrix layer has to have a threshold value $\mathbf{D}<\mathbf{X}_{\mathbf{T}}<2$ D below which the self-organization process starts. As $\mathbf{X}$ decreases below the threshold, the size of the uncovered areas in the matrix template increases and the amount of metal exposed to next metal arrival increases thus promoting $\mathrm{NCls}$ with larger diameter and consequently shorter length. The fact that the height of the NCls becomes smaller for $\mathbf{X}=0.5 \mathbf{D}$ (Fig. 3) supports this interpretation and provides a route to control the diameter of NCls. The maximum height of the $\mathrm{NCls}$ achievable by this method of production is therefore defined by the number of cycles and thus only limited by the stability of the production system.

In summary, the preparation of parallel Ag nanocolumns embedded in an oxide host by thin-film deposition technologies has been demonstrated, these features being essential for applications in integrated nanoelectronics or photonics. The number density, diameter and height of the nanocolumns can potentially be controlled by varying the amount of metal deposited in the first cycle (number of laser pulses in the metal target), the amount of metal deposited in subsequent cycles and the number of cycles, respectively. These results open new possibilities for material nano-engineering since they provide a simple route based on a single step process at room temperature to produce oriented metal nanocolumns with diameters typically $<10 \mathrm{~nm}$ and with low dimension and shape dispersion, features that are not easily accessible for other techniques.

This work was partially supported by TIC2002-03235, CICYT (Spain) and by ESP2002-04509-C04-01, and EU Network HPRN-CT-2002-00328. One of the authors (J.M.) acknowledges an I3P fellowship from the CSIC and the European Social Fund.

${ }^{1}$ E. C. Walter, R. M. Penner, H. Liu, K. H. Ng, M. P. Zach, and F. Favier, Surf. Interface Anal. 34, 409 (2002).

${ }^{2}$ G. Laurent, N. Félidj, J. Aubard, and G. Lévi, J. Chem. Phys. 122, 011102 (2005).

${ }^{3}$ D. H. Jeong, Y. X. Zhang, and M. Moskovits, J. Phys. Chem. B 108, 12724 (2004).

${ }^{4}$ J. L. Yao, Pure Appl. Chem. 72, 221 (2000).

${ }^{5}$ G. De Marzi, D. Iacopino, A. J. Quinn, and G. J. Redmond, Appl. Phys. (N.Y.) 96, 3458 (2004).

${ }^{6}$ Y. A. Pashkin, Y. Nakamura, and J. S. Tsai, Appl. Phys. Lett. 76, 2256 (2000).

${ }^{7}$ Y. J. Xiong, Y. Xie, C. Wu, J. Yang, Z. Li, and F. Xu, Adv. Mater. (Weinheim, Ger.) 15, 405 (2003).

${ }^{8}$ G. S. Metraux and C. A. Mirkin, Adv. Mater. (Weinheim, Ger.) 17, 412 (2005).

${ }^{9}$ V. Zielasek, T. Block, and H. Pfnur, Rev. Adv. Mater. Sci. 8, 1 (2004).

${ }^{10}$ Z. H. Wang, J. Liu, X. Chen, J. Wan, and Y. Qian, Chem.-Eur. J. 11, 160 (2004).

${ }^{11}$ C. Li, X. Yang, B. Yang, Y. Yan, and Y. Qian, Mater. Lett. 59, 1409 (2005).

${ }^{12}$ Y. Sakamoto, A. Fukuoka, T. Higuchi, N. Shimomura, S. Inagaki, and M. Ichikawa, J. Phys. Chem. B 108, 853 (2004).

${ }^{13}$ Y. Kondo and K. Takayanagi, Science 289, 606 (2000).

${ }^{14}$ M. P. Zach, K. H. Ng, and R. M. Penner, Science 290, 2120 (2000).

${ }^{15}$ W. Gotchy, K. Vonmetz, A. Leitner, and F. R. Aussenegg, Appl. Phys. B: Lasers Opt. 133, 381 (1996).

${ }^{16}$ See, for instance, Pulsed Laser Deposition, edited by D. B. Chrisey and G. K. Hubler (Wiley, New York, 1994); and C. N. Afonso, in Insulating Materials for Optoelectronics edited by F. Agulló-López (World Scientific, Singapore, 1995), Chap. 1, p. 1.

${ }^{17}$ J.-P. Barnes, A. K. Petford-Long, R. C. Doole, R. Serna, J. Gonzalo, A. Súarez-García, C. N. Afonso, and D. Hole, Nanotechnology 13, 465 (2002).

${ }^{18}$ J. Gonzalo, A. Perea, D. Babonneau, C. N. Afonso, N. Beer, J.-P. Barnes, A. K. Petford-Long, D. E. Hole, and P. D. Townsend, Phys. Rev. B 71, 125420 (2005).

${ }^{19}$ C. R. Henry, Surf. Sci. Rep. 31, 231 (1998).

${ }^{20}$ B. Thestrup, B. Toftmann, J. Schou, B. Doggett, and J. G. Lunney, Appl. Surf. Sci. 197, 175 (2002). 